\title{
Design of a Triangular Patch Microstrip Antenna on a Substrate of Photonic Crystal Material
}

\author{
Mandeep Singh \\ M. Tech Student
}

\author{
Juhi Rai \\ M. Tech Student \\ Department of Electronics and Communication \\ Sant Longowal Institute of Engineering and Technology, \\ Longowal, Punjab, India
}

\author{
Anupma Mrwaha \\ Associate Professor
}

\begin{abstract}
In this paper, a triangular patch microstrip antenna on the substrate material of a photonic crystal is designed and analyzed. The triangular shaped patch antennas are preferably used for research work because of its smaller coverage area attributes, however, similar to rectangular patch. The photonic crystal, due to band gap and periodicity of dielectric media is used as substrate for high gain, low return loss and reduction of back and side lobes. The designed antenna resonates at about $9 \mathrm{GHz}$ with return loss $S_{11}=-21.59 \mathrm{~dB}$ for the center location of the feed. The simulation has been performed by using high frequency structure simulator (HFSS) which is based on finite element method technique.
\end{abstract}

\section{General Terms}

Triangular patch microstrip antenna

\section{Keywords}

Triangular patch, photonic crystal, band gap, finite element method (FEM), HFSS.

\section{INTRODUCTION}

In recent years, the low profile microstrip patch antennas with various configurations have been developed for the extensive applications of wireless communication into both in the commercial sector and in the military sector. These patch antennas are widely used for the various applications due to its low cost, low profile, compatibility with IC technology, ease of fabrication, and ease of installation on the shaped surfaces. Particularly, triangular patches are preferred to design the antenna because they have the advantage of low cost and occupying smaller area for metallization on substrate than other configurations. The substrate of an antenna and the effect of radiation from the antenna play significant role for the analysis of electrical and physical characteristics of the designed antenna [1-3]. In the millimeter and microwave integrated circuits, use of high dielectric material as a substrate enhances the performance and functionality of the circuits. But then antenna radiates more efficiently towards the substrate than the air side and couples the power into surface-wave modes, due to which, power loss and edge currents are generated. The antenna efficiency is thus reduced and return losses are increased. If a thin substrate is used to avoid these losses then, a $180^{\circ}$ phase shift comes from the reflection at the base conductor, and causes the radiation cancel out at the observation point. So, if the substrate is replaced with the photonic crystal material whose photonic band gap encompasses the antenna excitation frequency, no surface modes and reduced back and side lobes are produced, because the power previously radiated into the substrate will be reflected towards the airside. By reducing the back and side lobes, the radiation pattern front-to-back ratio and overall antenna efficiency can be improved [4-8]. Photonic crystals have a class of periodic dielectric nanostructures in which the low dielectric constant air pillars are drilled equidistant from each other into high dielectric material in such a manner that electromagnetic wave propagation in all directions is completely prohibited for a range of frequencies which is called a "photonic band gap". The basic concept of this combination is to match the photonic band gap with the operational bandwidth of the patch antenna in order to reduce the effect of surface wave modes on the performance of the antenna and hence to improve the return loss, reduce the side lobe level and to solve the difficulties with the coupling consequently [9-12]. Agi and Malloy [13] have experimentally and computationally studied the integration of a microstrip patch antenna with a two-dimensional photonic crystal substrate.

In this paper, the design of a triangular microstrip patch antenna on photonic crystal material as substrate is presented. The patch antenna with and without photonic crystal are simulated and numerically analyzed using HFSS. The characteristic behavior of both antennas is compared to observe the optimal performance of a triangular patch antenna using $\mathrm{PC}$ as a substrate. The organization of the paper is as follows. Section 2 details the geometrical configuration of the triangular microstrip patch antenna. Section 3 is concerned with the design and principle of photonic crystal. In Section 4 the simulations are discussed and results obtained are investigated. Finally, Section 5 concludes the overall work.

\section{ANTENNA DESIGN}

The geometrical configuration of a triangular patch antenna is shown in Fig. 1. The patch having equal sides of $10.84 \mathrm{~mm}$ long is patterned on Rogers RO4003/Air substrate material with a dielectric constant of $\varepsilon_{r}=3.4$. The detailed geometrical parameters of patch antenna are shown in Table 1. The antenna is placed on the center of the substrate edge fed by a stripline conductor and operates at frequency of $10 \mathrm{GHz}$. A voltage signal of $1 \mathrm{~V}$ is applied to a transmission line with characteristic impedance $Z_{0}$ of $50 \Omega$ for single mode excitation of the microstrip patch using a lumped port. The quarter wavelength transformer (QWT) can be utilized to eliminate impedance mismatching. According to Ref. [14], the excitation of surface wave cannot be negligible if the ratio of the height and the wavelength of antenna $h / \lambda>0.03$. Therefore, at the working frequency, there must be a meaningful excitation of surface-wave modes for the thickness of the substrate considered for analysis. 


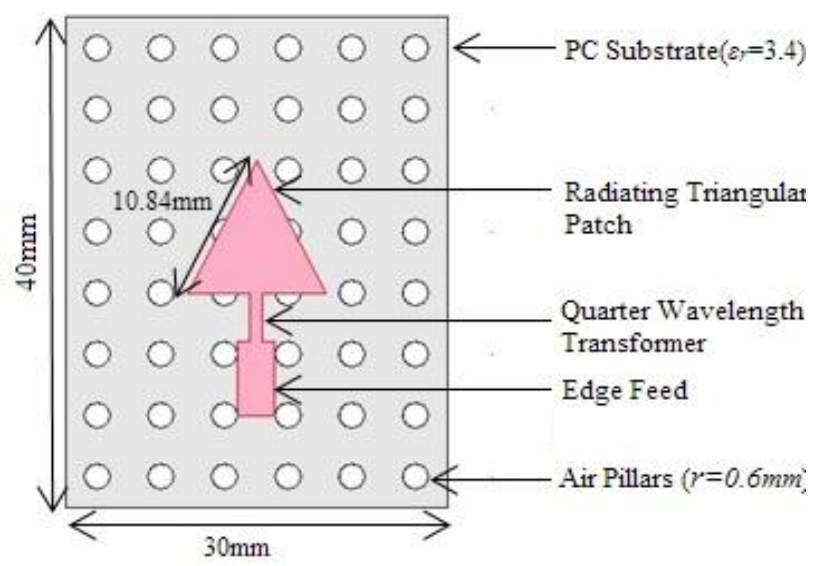

Fig. 1 The geometrical configuration of a triangular patch antenna

Table 1. Geometrical parameters of designed patch antenna

\begin{tabular}{|l|r|}
\hline Side length of triangular patch & $1.084 \mathrm{~cm}$ \\
\hline Width of edge feed & $0.35 \mathrm{~cm}$ \\
\hline Length of edge feed & $0.75 \mathrm{~cm}$ \\
\hline Length of $\lambda / 4$ transformer & $0.42 \mathrm{~cm}$ \\
\hline Width of $\lambda / 4$ transformer & $0.11 \mathrm{~cm}$ \\
\hline Height of substrate & $100 \mathrm{mil}$ \\
\hline
\end{tabular}

\section{PHOTONIC CRYSTAL DESIGN}

The conventional structure of photonic crystal, shown in fig. 2, is designed by drilling the air pillars with radius of $0.6 \mathrm{~mm}$ as low dielectric material into Rogers RO box as a high dielectric material having dimensions of $30 \mathrm{~mm} \times 40$ $\mathrm{mm} \times 100 \mathrm{mil}$. The photonic band gap is characterized by the radius of air pillars $r$, the lattice constant $a$, and the $r / a$ ratio [15]. By applying Plane Wave expansion method and considering the periodicity of dielectric media, the characteristic equation obtained from Maxwell's equations can be transformed into a standard diagonal matrix an eigenvalue problem. By solving this eigenvalue problem, the frequencies and the modes can be obtained [16]

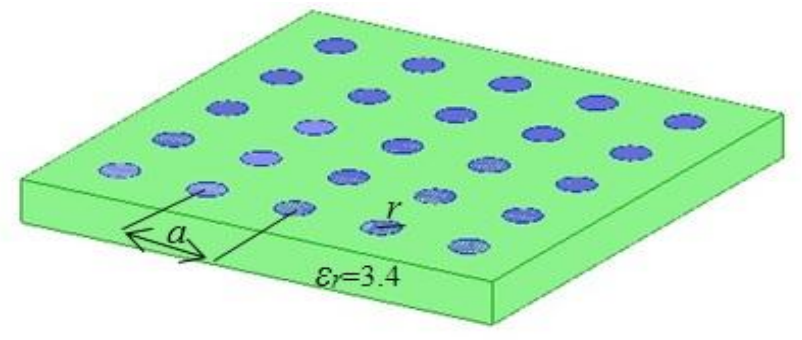

Fig.2 2-D Photonic crystal by a square lattice of air pillars drilled into a dielectric substrate

Generally, the band diagram for photonic crystal represents the relationship between the normalized frequency $f$ and the wavevector $k$. The curves along the wavevector path $\Gamma-\mathrm{X}-\mathrm{M}-\Gamma$ represent the frequency of the allowed propagation modes. The maximum gap between curves is photonic band gap through which no propagation exists. The wavevector path $\Gamma-\mathrm{X}-\mathrm{M}-\Gamma$ is the perimeter of the irreducible Brillouin Zone [17].
The inclusion of $\mathrm{PC}$ in the antenna design will provide the advantage of the reduction of surface wave losses caused by the radiation of power towards a dielectric media.

\section{SIMULATION AND ANALYSIS}

The software package Ansoft HFSS v.13 has been used for the simulation of triangular microstrip antenna as shown in fig.3. HFSS software is based on FEM which is a numerical technique to solve the partial differential equations representing the mathematical model of a 3-D structure. During simulation, FEM generates the meshed

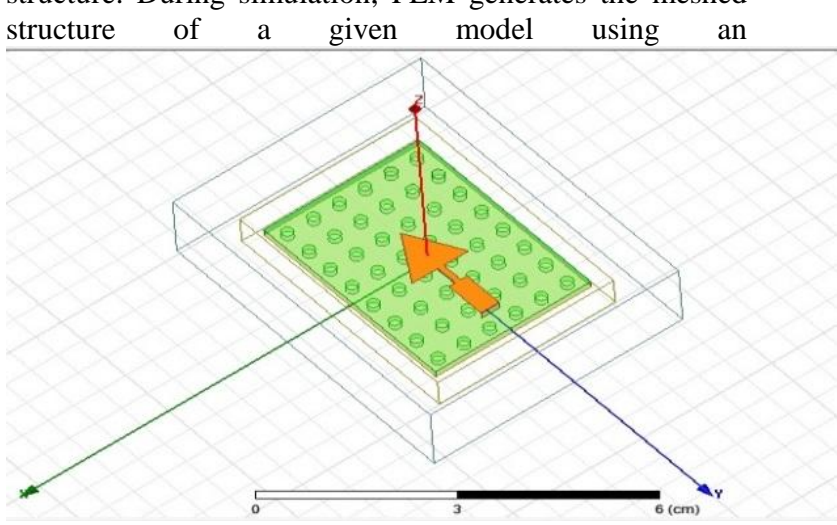

Fig. 3 HFSS model of a triangular patch antenna on photonic crystal as substrate

assemblage of triangular or quadrilateral shaped finite elements which together form the computational domain. The solution process generates the field variations which are utilized in post-processing for S-parameter determination and evaluation of radiation patterns.

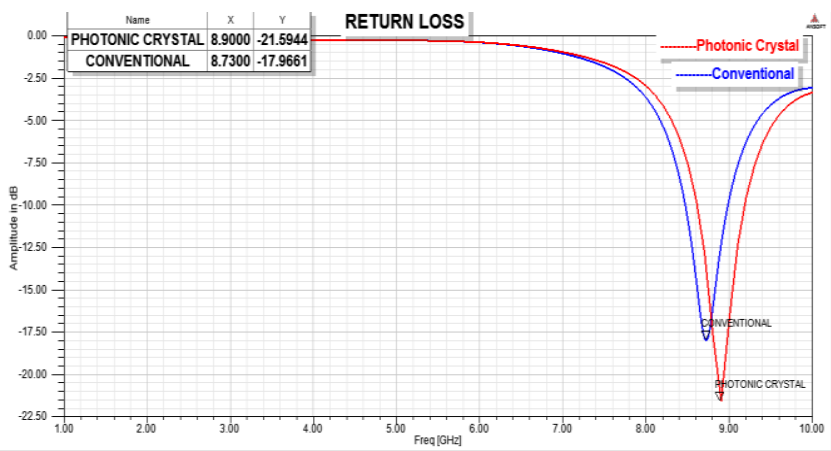

Fig.4 Comparative return losses of conventional and PC triangular patch antenna

Fig. 4 shows the return loss versus frequency plot for both configurations of antenna. It reveals that the return loss for $\mathrm{PC}$ antenna is below $-21.59 \mathrm{~dB}$ at the resonant frequency of about $9 \mathrm{GHz}$ as compared to a value of $-17.96 \mathrm{~dB}$ for the conventional patch antenna. The input return loss for antenna with photonic crystal is therefore lower than that for the antenna without photonic crystal and there is a small excursion of the resonance frequency because of the fringing fields from the sides of the patch. However, the dimensions of antenna can be changed by $2-4 \%$ in purpose to resonate exactly at $10 \mathrm{GHz}$. Here, the main concern is to analyze the radiation characteristics for optimized design of the triangular patch on photonic crystal hence neglecting minor deviations of resonant frequency. The $10 \mathrm{~dB}$ impedance bandwidth of the antenna is increased from $524 \mathrm{MHz}$ to $575 \mathrm{MHz}$ 


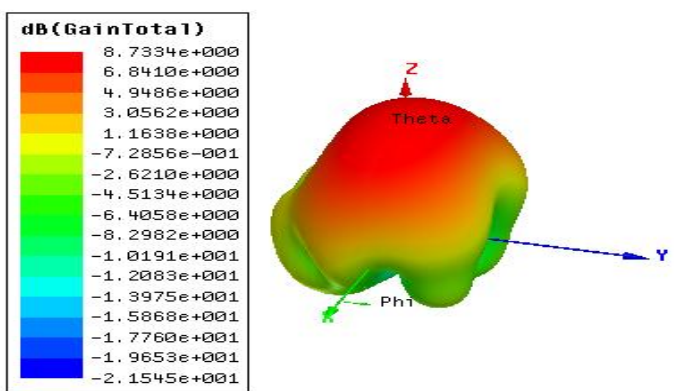

Fig. 5 3-D Radiation pattern of the designed photonic crystal antenna

In fig. 5, 3-D gain for the designed triangular patch antenna on photonic crystal as a substrate is plotted with the maximum gain of $8.7334 \mathrm{~dB}$.

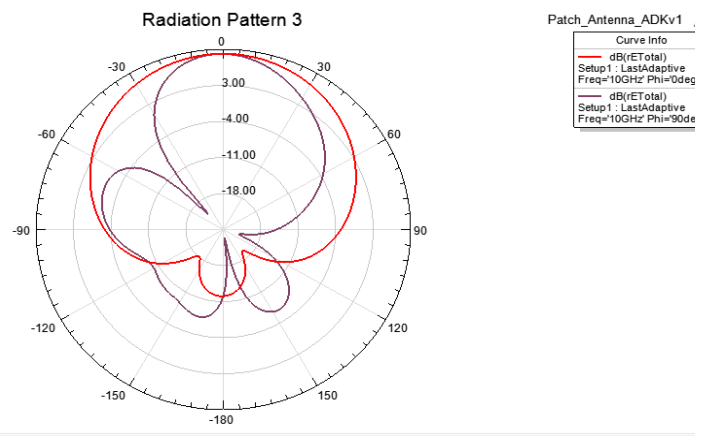

(a) Conventional antenna

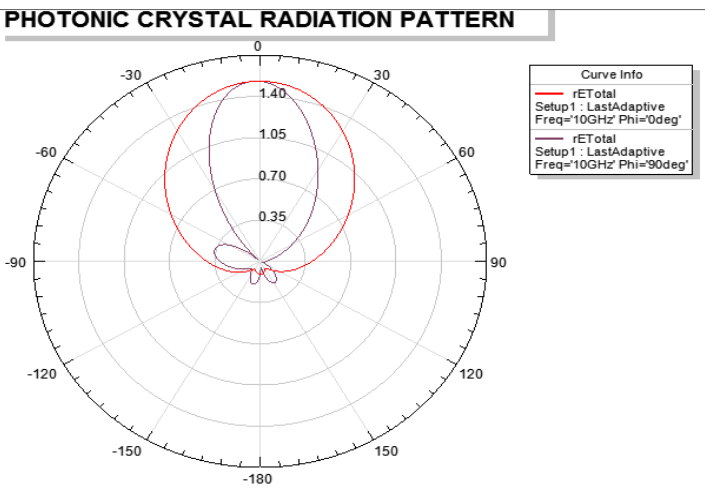

(b) Photonic crystal Antenna Fig. 6 Far-field radiation pattern

The far field radiation patterns for both antenna models are depicted in fig. 6. It can be seen from fig. 6(a), that the radiation pattern includes high back and side lobes for the conventional patch antenna which get reduced significantly for the photonic crystal antenna shown in fig. 6(b), as the inclusion of photonic crystal as a substrate reduces the excitation of the surface wave modes.

The output parameters of the designed triangular patch antenna on photonic crystal substrate are shown in Table 2.
Table 2. Output parameters of a PC antenna

\begin{tabular}{|l|r|}
\hline Gain & $8.7334 \mathrm{~dB}$ \\
\hline Return Loss & $-21.59 \mathrm{~dB}$ \\
\hline Bandwidth & $575 \mathrm{MHz}$ \\
\hline Directivity & $7.28 \mathrm{dBi}$ \\
\hline
\end{tabular}

\section{CONCLUSIONS}

The paper implements the concept of design of triangular microstrip patch antenna using photonic crystal material as substrate. At the operating frequency, the return loss, directivity, , and the gain of a printed circuit antenna can be efficiently improved with reduced back lobe level by the reduction or, in some cases, elimination of surface waves and by changing the geometrical shapes and configuration of patch on PC substrate. Using the air pillars in high dielectric materials, promising results have been obtained by through numerical simulation using Ansoft HFSS. The results suggest the method to solve the problem of degradation in performance by the elimination of the surface wave modes in the design of devices with higher dielectric constant and thicker substrate.

\section{REFRENCES}

[1] Ma S L, Row J S. "Design of single-feed dualfrequency patch antenna for GPS and WLAN applications". IEEE Transactions on Antennas and Propagation, Vol. 59, No. 9, 3433-3436, 2011.

[2] Singh V K, "Ka-band micro machined microstrip patch antenna". IET Microwaves Antennas \& Propagation, Vol. 4, No. 3, 316-323, 2010.

[3] Sharma, A. and G. Singh, "Rectangular microstrip patch antenna design at $\mathrm{THz}$ frequency for communication systems", Int. J. Infrared and Millimeter Waves, (under review), Dec. 2007.

[4] Ozbay, E., B. Temelkuran, and M. Bayindir, "Microwave applications of photonic crystals", Progress In Electromagnetics Research, PIER 41, 185-209, 2003.

[5] Yang, H. Y. D., N. G. Alexopoulos, and E. Yablonovitch, "Photonic band gap materials for high gain printed circuit antennas", IEEE Trans. Antenna and Propagation, Vol. 45, 185-187, 1997.

[6] Meade, R. D., K. D. Brommer, A. M. Rappe, and J. D. Joannapoulos, "Photonic band states in periodic dielectric materials", Phys. Rev. B: Condensed Matter, Vol. 44, 13772-13774, 1991.

[7] Coccioli, R., W. R. Deal, and T. Itoh, "Radiation characteristics of a patch antenna on a thin PBG substrate", IEEE Trans. Antennas Propag., Vol. 45, 656-659, 1998.

[8] Gonzalo, R. and B. Martinez, "The effect of dielectric permittivity on the properties of photonic band gap devices", Microwave and Optical Technology Lett., Vol. 23, No. 2, 92-95, 1999.

[9] Radisic, V., Y. X. Qian, R. Coccioli, and T. Itoh,, "Novel 2D photonic bandgap structure for microstrip antenna", IEEE Microwave and Guided Wave Lett., Vol. 8, 69-71, 1998. 
[10] Fernandes, H. C. C. and A. R. B. da Rocha, "Analysis of antennas with PBG substrate", Int. J. Infrared and Millimeter Waves, Vol. 24, No. 7, 1171-1176, 2003.

[11] Gonzalo R, Demaagt P, Mario S. "Enhanced patch antenna performance by suppressing surface wave using photonic crystal substrates", IEEE Trains Microwave Theory and Techniques, Vol. 47, No. 11,2131-2138, 1999.

[12] Ho, K. M., C. T. Chan, and C. M. Soukoulish, "Existence of a photonic gap in periodic dielectric structures", Phys. Rev. Lett., Vol. 65, 3152-3155, 1990.

[13] Agi, K. and J. Malloy, "Integration of a microstrip patch antenna with a two dimensional photonic crystal substrate", Electromagnetics, Vol. 19, 277290, 1999.
[14] James J R, Henderson A, "High-frequency behavior of microstrip open-circuit terminations. Microwave", Optics and Acoustics, Vol. 3, No. 5, 205-218, 1979.

[15] J D Joannapolous, R D Meade and J N Winn, "Photonic crystals: Molding the flow of light", Princeton University Press, New Jersey 08540, 1995.

[16] Zhang, Z .and S. Satpathy, "Electromagnetic wave propagation in periodic structures: Bloch wave solution of Maxwell equations", Physical Rev. Lett., Vol. 65, 2650-2653, 1990.

[17] YEE, K. "Numerical solution of initial boundary value problems involving Maxwell's equations in isotropic media”, IEEE Trans. Antennas Propag., vol. 14, no. 3, 302-307, 1966. 